\title{
Rotation-Induced Mixing in Red Giant Stars
}

\author{
Ana Palacios ${ }^{1}$, Corinne Charbonnel ${ }^{2,3}$, Suzanne Talon ${ }^{4}$, and Lionel Siess ${ }^{1}$ \\ 1 IAA-Université Libre de Bruxelles, CP-226 Bd du Triomphe, B-1050 Brussels, \\ Belgium, \\ 2 Geneva Observatory, 51 chemin des Maillettes, CH-1290 Sauverny, Switzerland \\ 3 LA2T-OMP, UMR 5572, 14 av. E. Belin, F-31400 Toulouse, France \\ 4 Département de Physique, Université de Montréal, Montréal PQ H3C 3J7, Canada
}

\section{Introduction}

Red giant stars, both in the field and in globular clusters, present abundance anomalies that can not be explained by standard stellar evolution models. Some of these peculiarities, such as the decline of ${ }^{12} \mathrm{C} /{ }^{13} \mathrm{C}$, and that of $\mathrm{Li}$ and ${ }^{12} \mathrm{C}$ surface abundances for stars more luminous than the bump, clearly point towards the existence of extra-mixing processes at play inside the stars, the nature of which remains unclear. Rotation has often been invoked as a possible source for mixing inside Red Giant Branch (RGB) stars ([8],[1],[2]). In this framework, we present the first fully consistent computations of rotating low mass and low metallicity stars from the Zero Age Main Sequence (ZAMS) to the upper RGB.

\section{Physics of the Models}

We present three models of a $0.85 \mathrm{M}_{\odot}, \mathrm{Z}=10^{-5}$ star. Model $\mathbf{A}$ is a standard non-rotating model. Model $\mathbf{B}$ is a slowly rotating model with $v_{\text {ZAMS }}=5 \mathrm{~km} . \mathrm{s}^{-1}$, undergoing no magnetic braking, and for which we assumed a solid body rotating convective envelope (CE) $\left(\Omega_{\mathrm{CE}}=\mathrm{cst}\right)$ during the whole evolution. Model $\mathbf{C}$ is an initially rapidly rotating model with $v_{\text {ZAMS }}=110 \mathrm{~km} \cdot \mathrm{s}^{-1}$, which undergoes magnetic braking $\left(v_{\mathrm{TO}}=3 \mathrm{~km} \cdot \mathrm{s}^{-1}\right)$, and for which we assumed uniform specific angular momentum in the $\mathrm{CE}\left(\Omega_{\mathrm{CE}} \propto \mathrm{r}^{-2}\right)$. In our rotating models, we compute the transport of both angular momentum and chemicals by meridional circulation and shear-induced turbulence from the ZAMS on, according to [5]. We use the new prescription for the horizontal turbulent viscosity given by [6].

\section{Main Results}

Rotational mixing does not affect significantly the stellar structure $\left(\mathrm{L}_{\text {bump }}\right.$ is the same in models $\mathbf{A}, \mathbf{B}$ and $\mathbf{C}$ ), but leads to larger abundance variations on the lower RGB associated with a deeper first dredge-up (Fig. 1b).

When solid body rotation is assumed in the CE (model $\mathbf{B}$ ), the degree of differential rotation at its base is too low to trigger efficient shear-induced turbulence between the outer part of the hydrogen burning shell (HBS) and the CE (solid lines in Fig. 1a). On the contrary, in our model $\mathbf{C}$ the differential rotation 
A. Palacios et al.
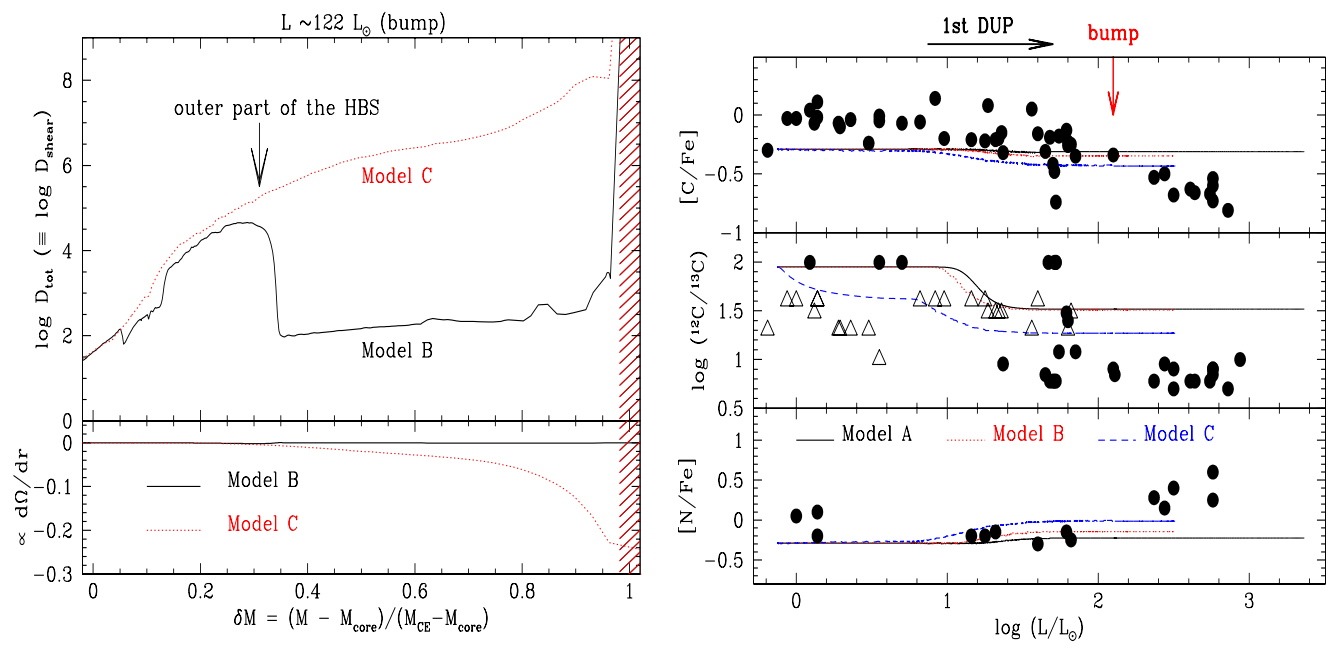

Fig. 1. (a) (left) Profiles at the bump, of the total diffusion coefficient (top) and of the degree of differential rotation (bottom) for model $\mathbf{B}$ (solid lines) and model $\mathbf{C}$ (dotted lines). Hatched regions correspond to the CE. (b) (right) Comparison of our models with observations ([4]). Triangles are lower limits. Dots are actual values.

of the $\mathrm{CE}$ ensures the onset of turbulence in the contiguous radiative region, and the $\mathrm{CE}$ is connected to the outer HBS through shear mixing (dotted lines in Fig. 1a). This confirms the conjecture by [8], [7] and [2] that the shear-mixing efficiency is enhanced in models with differentially rotating $\mathrm{CE}$.

In our most favorable case (model $\mathbf{C}$ ), the maximum value of the diffusion coefficient in the outer part of the HBS (where abundances of Li, C and $\mathrm{N}$ present large variations) is $10^{5} \mathrm{~cm}^{2} \cdot \mathrm{s}^{-1}$ (Fig. 1a), far from the $410^{8} \mathrm{~cm}^{2} \cdot \mathrm{s}^{-1}$ value that seems to be necessary to reproduce the observations according to [3]. As a result none of our rotating models can reproduce the observed patterns emphasized by [4] (Fig. 1b).

Rotation remains the best candidate for extra-mixing in RGB stars. The present modelling of the rotational mixing is however still incomplete, and agreement between self-consistent models and observations might be achieved by improving the description of the hydrodynamics related to rotation.

\section{References}

1. C. Charbonnel: ApJL 453, L41 (1995)

2. P.A. Denissenkov, C.A. Tout: MNRAS 399, 603 (2000)

3. P.A. Denissenkov, D.A. VandenBerg: ApJ 593, 509 (2003)

4. R. G. Gratton , C. Sneden , E. Carretta , A. Bragaglia: A\&A 354, 169 (2000)

5. A. Maeder, J.-P. Zahn: A\&A 334, 1000 (1998)

6. S. Mathis, A. Palacios, J.-P. Zahn: A\&A 425, 243 (2004)

7. A. Sills, M. Pinsonneault: ApJ 540, 489 (2000)

8. A.V. Sweigart, J.G. Mengel: ApJ 229, 624 (1979) 